\title{
MiRNA expression profiles reveal the involvement of miR-26a, miR-548I and miR-34a in hepatocellular carcinoma progression through regulation of ST3GAL5
}

\author{
Hongjiao Cai ${ }^{1,2,4}$, Huimin Zhou ${ }^{3,4}$, Yuan Miao ${ }^{1}$, Nana Li ${ }^{1}$, Lifen Zhao ${ }^{1}$ and Li Jia ${ }^{1}$
}

MicroRNAs (miRNAs) have key roles in comprehensive physiological and pathological processes by targeting specific genes through translational repression. Identification of miRNAs related to metastasis enables us to obtain better insight into cancer development. In the current study, we investigated the miRNA expressional profiles in the highly invasive human hepatocellular carcinoma cell line MHCC97-H and MHCC97-L with lower metastatic potential using miRNA microarrays. By quantitative real-time PCR, we confirmed the results of miRNA experiments. Thirteen differentially expressed miRNAs were identified between MHCC97-H and MHCC97-L cells; and the same results were found in clinical samples. Using bioinformatic analysis and luciferase reporter assay, we found that ST3GAL5, a sialyltransferase gene, was the direct target of miR-26a, miR-548I and miR-34a. Engineered expression of miR-26a, miR-548I or miR-34a in MHCC97-H or MHCC97-L cells could significantly change their malignant behaviors and oncogenicity in in vitro and in vivo assays. Manipulated expression of ST3GAL5 also led to the alteration of the metastatic potential of MHCC97-H and MHCC97-L cells, in agreement with the effects of above three miRNAs. Altogether, our data indicate that the levels of these miRNAs may be used as biological markers for evaluating hepatocellular carcinoma progression. miR-26a, miR-548I and miR-34a, acting as tumor suppressors, may exert their effects by regulating ST3GAL5.

Laboratory Investigation (2017) 97, 530-542; doi:10.1038/labinvest.2017.12; published online 20 February 2017

Liver cancer, one of the most common malignancies, caused 358840 cases and 312432 deaths in China in 2010. ${ }^{1}$ In 2012, there were about 782500 new liver cancer cases and 745500 deaths occurred worldwide, with China alone accounting for nearly $50 \%$ of the total number of cases and deaths. ${ }^{2}$ Most (70-90\%) primary liver cancers occurring worldwide are hepatocellular carcinoma (HCC). ${ }^{3}$ The 5 -year survival rate of patients is $<30 \%$ due to the high recurrence and metastasis rate, ${ }^{4}$ which is the leading cause of death in patients with HCC.

Metastasis is the most lethal attribute of a tumor. Numerous genes and molecules have been shown to be associated with the metastatic cascade. ${ }^{5}$ In recent years, development of gene chip technology has opened a new era in the field of tumor biology. MiRNA microarray technology can be applied as a screening tool for differentially expressed miRNAs in cancer cells and can also provide insight into the mechanisms of oncogenesis and metastasis of tumors.
MiRNAs are a class of small, endogenous, single-stranded, noncoding RNAs that have important regulatory roles in animals and plants by targeting mRNAs through translational repression or cleavage. ${ }^{6}$ MiRNAs are involved in cellular processes, such as inflammation, cell cycle regulation, stress response, differentiation, apoptosis and migration. ${ }^{7}$ To date, a number of miRNAs have been implicated in human cancers. Shao $e$ t $a l^{8}$ reported that miR-26a and miR-144 could inhibit cell proliferation and metastasis in esophageal squamous cell carcinoma by regulating COX-2. Qiu et al ${ }^{9}$ showed that four miRNAs (miR-26a, miR-142-3p, miR-148a and miR-195) wree downregulated in the tissue and plasma of the gastric cancer patients compared with controls by using Agilent Human miRNA Microarray and TaqMan low density array and receiver operating characteristic curves, which revealed that miR-26a had the highest diagnostic accuracy. In another report, miR-5481 was found to suppress migration and invasion of non-small cell lung cancer (NSCLC) cells,

\footnotetext{
${ }^{1}$ College of Laboratory Medicine, Dalian Medical University, Dalian, Liaoning Province, China; ${ }^{2}$ Department of Central Laboratory, Dalian Municipal Central Hospital, Dalian, Liaoning Province, China and ${ }^{3}$ Department of Microbiology, Dalian Medical University, Dalian, Liaoning Province, China

Correspondence: Dr. L Jia, College of Laboratory Medicine, Dalian Medical University, 9 Lvshunnan Road Xiduan, Dalian, Liaoning Province 116044, China.

E-mail: jiali0081@sina.com

${ }^{4}$ These authors contributed equally to this work.

Received 6 September 2016; revised 24 December 2016; accepted 13 January 2017
} 
negatively regulating lymph node metastasis of NSCLC. ${ }^{10}$ It has been shown in the literature that aberrant miRNA expression may also affect metastasis, subtype classification and prognosis of HCC cells, ${ }^{11,12}$ implying that miRNAs are potential biomarkers and therapeutic targets for HCC patients.

Glycosylation is one of the most important modifications of proteins and lipids and is catalyzed by glycosyltransferases (GTs). In recent years, alteration in glycosylation and its relationship to carcinogenesis and development have been explored intensively. ${ }^{13}$ Among GTs, the sialyltransferases (ST) are a family of enzymes that transfer sialic acid residues from an activated CMP-sialic acid donor to the termini of either $\mathrm{N}$ - or O-linked glycans of glycoprotein or glycolipids. The ST family consists of four subfamilies (ST3GalI-VI, ST6GalI-II, ST6GalNAcI-VI, ST8SiaI-VI). ${ }^{14}$ Aberrant ST levels are found in a number of types of human and experimental cancers. It has been reported that ST6Gal I and ST3Gal V mRNAs were increased in lymphoblasts of acute lymphoblastic leukemia patients, whereas its presence was negligible in non-malignant donors. These two genes were also shown to be positively correlated with a high risk of the disease. ${ }^{15}$ Pérez-Garay et al ${ }^{16}$ demonstrated that ST3Gal III modulated motility and adhesion of pancreatic cancer cells in vitro, while the overexpression of ST3Gal III enhanced cell metastatic potential in vivo. Although abnormal levels of STs are often observed in cancer cells, their upstream or downstream regulators have been explored in very few studies.

In the present study, we analyzed comprehensive miRNA profiles and identified differentially expressed miRNAs in HCC cells with different metastatic potentials by miRNA microarray analysis. By quantitative real-time PCR (qRTPCR), we confirmed the results of miRNA chip studies in HCC cells and clinical samples. In vitro and in vivo functional tests indicated that miR-26a, miR-5481 and miR-34a negatively mediated the malignant behavior of HCC cells. In addition, for the first time, we identified that ST3GAL5, a glycotransferase gene, was the direct target for miR-26a, miR-5481 and miR-34a regulation at the $3^{\prime}$-UTR in HCC cell lines. These results support the hypothesis that miRNAs and their downstream genes are associated with metastasis of HCC cells. Further analysis of these miRNAs will help us to understand the potential mechanisms of HCC development.

\section{MATERIALS AND METHODS Cell Lines}

Two HCC cell lines, MHCC97-H and MHCC97-L (of the same genetic background but with high and low metastatic potential were previously generated from the parental HCC cell line MHCC97) were obtained from the KeyGEN (Nanjing, China) and kept in Dulbecco Modified Eagle's Medium (Gibco, Grand Island, NY, USA) with 10\% heatinactivated fetal bovine serum (Gibco). All cells were fostered in a humidified atmosphere of $5 \% \mathrm{CO}_{2}$ at $37^{\circ} \mathrm{C}$.

\section{Cell Transfection}

To modulate miRNA and ST3GAL5 expression, HCC cells at $50 \%$ confluency were incubated with various concentrations of mimic and inhibitor (10, 50, 100 and $200 \mathrm{nM}$ ) to determine the maximum effective concentration, by western blotting analysis, which was $100 \mathrm{nM}$. Then cells were grown in antibiotic-free media and were transfected with Pre$\mathrm{miR}-26 \mathrm{a} / \mathrm{miR}-5481 / \mathrm{miR}-34 \mathrm{a} / \mathrm{mixture}$ mimics or an anti-miR$-26 \mathrm{a} / \mathrm{miR}-548 \mathrm{l} / \mathrm{miR}-34 \mathrm{a} / \mathrm{mixture}$ and ST3GAL5 or shRNA against ST3GAL5, provided by Shanghai GenePharma, (Shanghai, China) at a final concentration of $100 \mathrm{nM}$ and $50 \mathrm{nM}$, respectively, using Lipofectamine 2000 Transfection Reagent (Invitrogen) according to the manufacturer's instructions. At $48-72 \mathrm{~h}$ after transfection, cells were collected and miRNA levels were detected by qRT-PCR.

\section{Tissue Specimens}

Twenty-seven HCC tissues and relevant adjacent tissues $(3 \mathrm{~cm}$ from the tumor edge) were collected from patients who underwent surgical resections from June 2013 to April 2015 at the First Affiliated Hospital of Dalian Medical University, with the informed consent of the patients, and the experiments were approved by the Ethics Committee of the First Affiliated Hospital of Dalian Medical University. The patients included 8 men and 19 women, with an age range from 19 to 78 years, and the mean age was 51.3 years. All patients never received any radiation or chemotherapy treatment before the surgery. All cases have been confirmed by pathological diagnosis and were staged according to the 2012 HCC staging system of the International Union against Cancer. Samples were snap-frozen in liquid nitrogen after collection. The clinico-pathological features of the patients are summarized in Table 1.

\section{Animal Models}

Female athymic nude mice, aged 5 weeks, $15-20 \mathrm{~g}$ in weight, were purchased from the Animal Research Center of Dalian Medical University. All mice were maintained in specificpathogen-free conditions, and all animal protocols were approved by the Dalian Medical University Committee on Animal Research.

\section{RNA Extraction and qRT-PCR Analysis}

According to the manufacturer's protocol, TRIzol (Invitrogen, Carlsbad, CA, USA) and RNeasy Mini Kit (QIAGEN, Valencia, CA, USA) were used to isolate and purify total RNA from HCC cell lines and frozen tissues. The Nanodrop spectrophotometer (ND-1000, Nanodrop Technologies) was used to measure RNA quality and quantity, and gel electrophoresis was used to determine RNA integrity. QuantiTect Reverse Transcription Kit (QIAGEN) was used to synthesize cDNA, and the expression levels of miRNAs were detected by using the mirVanaTM qRT-PCR microRNA Detection Kit (Ambion, Austin, TX, USA) on a Biosystems 7300 Real-Time PCR system (ABI, Foster City, CA, USA). All data were analyzed using the $2^{-\Delta \Delta C T}$ method and normalized to U6-small nuclear RNA. 
Table 1 Clinico-pathological characteristics of HCC patients

\begin{tabular}{|c|c|}
\hline Characteristics & $n$ \\
\hline \multicolumn{2}{|l|}{ Group } \\
\hline Cancer tissues & 27 \\
\hline Transitional tissues & 27 \\
\hline \multicolumn{2}{|l|}{ Gender } \\
\hline Male & 19 \\
\hline Female & 8 \\
\hline \multicolumn{2}{|l|}{ Age (years) } \\
\hline$\geq 50$ & 17 \\
\hline$<50$ & 10 \\
\hline \multicolumn{2}{|l|}{ HBV infection } \\
\hline Negative & 6 \\
\hline Positive & 21 \\
\hline \multicolumn{2}{|l|}{ Serum AFP level $(\mu g / l)$} \\
\hline$\geq 200$ & 18 \\
\hline$<200$ & 9 \\
\hline \multicolumn{2}{|l|}{ Distant metastasis } \\
\hline No & 27 \\
\hline \multicolumn{2}{|l|}{ Lymph node metastasis } \\
\hline Absent & 27 \\
\hline \multicolumn{2}{|l|}{ Clinical stage } \\
\hline$|-| \mid$ & 27 \\
\hline
\end{tabular}

AFP, alpha-fetoprotein; HBV, hepatitis B virus; HCC, Hepatocellular carcinoma.

\section{MiRNA Labeling and Array Hybridization}

RNA labeling and array hybridization was commissioned by KangCheng, according to Exiqon's manual. After quality control, the miRCURY Hy3/Hy5 Power Labeling Kit (Exiqon, Vedbaek, Denmark) was used according to the manufacturer's guideline for miRNA labeling by the following steps: First, $1 \mu \mathrm{l}$ RNA in $2.0 \mu \mathrm{l}$ of water was combined with $1.0 \mu \mathrm{l}$ of CIP buffer and CIP (Exiqon). The mixture was incubated for $30 \mathrm{~min}$ at $37^{\circ} \mathrm{C}$. Second, the reaction was terminated by incubation for $5 \mathrm{~min}$ at $95^{\circ} \mathrm{C}$. Then $3.0 \mu \mathrm{l}$ of labeling buffer, $1.5 \mu \mathrm{l}$ of fluorescent label (Hy3TM), $2.0 \mu \mathrm{l}$ of DMSO and $2.0 \mu \mathrm{l}$ of labeling enzyme were added to the mixture. The labeling reaction was incubated for $1 \mathrm{~h}$ at $16^{\circ} \mathrm{C}$. The reaction was terminated by incubation for $15 \mathrm{~min}$ at $65^{\circ} \mathrm{C}$. After stopping the labeling procedure, the Hy3-labeled samples were hybridized on the miRCURYTM LNA Array (v. 18.0) (Exiqon) according to the array manual. The total $25 \mu \mathrm{l}$ mixture from Hy3-labeled samples with $25 \mu$ l hybridization buffer were first denatured for $2 \mathrm{~min}$ at $95^{\circ} \mathrm{C}$, incubated on ice for $2 \mathrm{~min}$ and then hybridized to the microarray for $16-20 \mathrm{~h}$ at $56^{\circ} \mathrm{C}$ in a 12-Bay Hybridization Systems (Hybridization System-Nimblegen Systems, Madison, WI, USA). Following hybridization, the slides were achieved and washed several times using the Wash Buffer Kit (Exiqon) 3. Then the slides were scanned using the Axon GenePix 4000B microarray scanner (Axon Instruments, Foster City, CA).

\section{Target Gene Prediction}

Target genes of miRNAs were predicted by three major online miRNA target prediction algorithms: miRanda (http://www. microrna.org/microrna/home.do), microCosm (http://www.ebi. ac.uk/enright-srv/microcosm/htdocs/targets/v5/), and TargetScan (http://www.targetscan.org).

\section{Luciferase Assays}

HEK 293 T cells were seeded in a 24 -well dish $\left(5 \times 10^{4}\right.$ cells per well) at 50\% confluence, and wild-type or mutant ST3GAL5-3'-UTR pmirGLO plasmids were constructed by using pmirGLO Dual-Luciferase miRNA Target Expression Vector (Promega, Madison, WI, USA). These plasmids were co-transfected with miRNA mimics or miR-NC into HEK 293T cells using Lipofectamine 2000 Transfection Reagent (Invitrogen). Luciferase activity was measured at $48 \mathrm{~h}$ posttransfection by using the Dual-Luciferase Reporter Assay (Promega) according to the manufacturer's instructions.

\section{Western Blotting Analysis}

Cells were harvested after treatment and total protein was extracted, equal amount of protein was separated by electrophoresis on 10\% SDS-PAGE (sodium dodecyl sulfatepolyacrylamide gel) and then transferred onto polyvinylidene difluoride membrane (Pall Corporation). The membrane was blocked in PBS with 0.1\% Tween 20 (PBST) and 5\% powdered skim milk for an hour at room temperature, and the membrane was then incubated with anti-ST3GAL5 monoclonal antibody (Abcam, Cambridge, UK, 1:1000 dilution) overnight at $4{ }^{\circ} \mathrm{C}$ and horseradish peroxidase (HRP)-conjugated secondary antibodies. Signal was detected using the ECL Western Blotting Kit (Amersham Biosciences, UK). GAPDH was used as a control and detected signal was normalized to GAPDH.

\section{Cell Proliferation Assay}

Cell Counting Kit-8 (CCK-8; KeyGEN) was used for the cell proliferation assays. Cells $\left(1 \times 10^{3} /\right.$ well $)$ were seeded in 96-well plates with $100 \mu \mathrm{l}$ of DMEM medium containing $10 \%$ FBS. The plates were put in a humidified incubator for 24,48 and $72 \mathrm{~h}$. At the indicated time points, $10 \mu \mathrm{l} \mathrm{CCK-8}$ solution was added and then incubated at $37^{\circ} \mathrm{C}$ for $2 \mathrm{~h}$. The 
absorbance at $450 \mathrm{~nm}$ was measured with a microplate reader to detect the number of viable cells.

\section{Colony-Forming Assay}

Cells prepared for assay were trypsinized for $10 \mathrm{~min}$ and resuspended, $500 \mu \mathrm{l}$ cells $\left(1 \times 10^{3} / \mathrm{ml}\right)$ were seeded into sixwell plates and to each well was added $1.5 \mathrm{ml}$ DMEM (containing $10 \% \mathrm{FBS}$ ). The plates were incubated at $37^{\circ} \mathrm{C}$, $5 \% \mathrm{CO}_{2}$, and the medium was changed every 3 days until conspicuous colonies were observed. Then colonies were stained with crystal violet and counted.

\section{Cell Migration and Invasion Assay}

A transwell assay was used to evaluate the migratory and invasive potential of cancer cells. First gelatin and Matrigel were coated on filter to form a continuous thin layer, then the single-cell suspension was added into the upper chamber (before the experiments, cells were incubated for $24 \mathrm{~h}$ in serum-free media) and to the lower chamber $500 \mu \mathrm{l}$ DMEM medium containing 10\% FBS was added. The cells were placed in an incubator for $24 \mathrm{~h}$ with a humidified atmosphere of $5 \% \mathrm{CO}_{2}$ at $37^{\circ} \mathrm{C}$. When the chamber was taken out, the filter was fixed with $4 \%$ formaldehyde and stained with Wright Giemsa. The migratory and invasive cells were counted and photographed in 10 different fields of each filter.

\section{Tumor Xenograft Models}

Five-week-old female athymic nude mice were used to establish animal models. Cultured cancer cells (approximately $1 \times 10^{7}$ ) were respectively injected subcutaneously into the right flank of each nude mouse. One week later, the mice were injected intratumorally with the miR-26a/miR-548l/ $\mathrm{miR}-34 \mathrm{a} / \mathrm{mixture} /$ control mimics or miR-26a/miR-5481/miR$-34 \mathrm{a} /$ mixture/control inhibitors three times per week for 3 weeks, respectively. The tumor size was monitored and measured by vernier calipers weekly, and the volume of the tumor was calculated by the following formula: Tumor volume $=1 / 2$ (length $\times$ width $\left.^{2}\right)$. Four weeks later, mice were killed and the tumors were stripped, weighed, photographed and recorded.

\section{Immunohistochemical (IHC) Analysis}

The tumors were dissected and fixed with $4 \%$ paraformaldehyde and then dehydrated in gradient concentrations of alcohol before paraffin embedding. The tissues were sliced into $5 \mu \mathrm{m}$ sections, then the sections were dried, deparaffinized, rehydrated and immersed in 3\% hydrogen peroxide for 10 min for blocking endogenous peroxidase. The slices were washed with PBS, ST3GAL5 antibody was added, incubated at $4{ }^{\circ} \mathrm{C}$ overnight and then the slices were stained with streptavidin-HRP-conjugated antibody for $1 \mathrm{~h}$ at room temperature and counterstained with hematoxylin. Finally, the expression of protein was analyzed by the Image-ProPlus 4.5 Software (Media Cybernetics, USA).

\section{Statistical Analysis}

All data were presented as mean \pm s.d., and the comparison of two groups were carried out using Student's $t$-tests. $P$-value $<0.05$ was considered statistically significant. Statistical analysis was performed using SPSS 17. 0 statistical packages (SPSS, Chicago, IL, USA).

\section{RESULTS}

\section{Identification of HCC-Related miRNAs}

To explore the relationship between miRNAs and HCC metastasis, we performed miRNA microarray analysis to examine the global miRNA expression in MHCC97-H and MHCC97-L cell lines. The results show that 13 differentially expressed miRNAs were found (fold change >2.0) in MHCC97-H cells compared with MHCC97-L cells. These 13 miRNAs were selected using $P<0.05$ as cutoff and included 5 upregulated miRNAs (miR-1284, miR-542-3p, miR-877-3p, miR-643, miR-3943) and 8 downregulated miRNAs (miR-377-5p, miR-5481, miR-146b-5p, ebv-miRBARTA19-3p, miR-26a-2-3p, miR-455-3p, miR-34a-5p, miR-146a-5p) (Table 2). The map of unsupervised hierarchical clustering analysis clearly showed the distinct miRNA expression levels between MHCC97-H and MHCC97-L cells (Figure 1). The results indicated that miRNAs may be involved in HCC progression. To validate the microarray data (five upregulated and eight downregulated genes), qRT-PCR was conducted to detect the differential miRNA expression levels in cell lines and tissues. Compared with MHCC97-L cell lines, miR-1284 (2. 79-fold), miR-542-3p (2.68-fold), miR-877-3p (3.02-fold), miR-643 (4.98-fold) and miR-3943 (1.84-fold) expression were significantly higher and miR-377$5 \mathrm{p}$ (2.35-fold), miR-548l (3.31-fold), miR-146b-5p

Table 2 Different miRNA expression in MHCC97-H cells and MHCC97-L cells

\begin{tabular}{lcll}
\hline miRNA & Fold change (H vs L) & $P$-value & Up/down \\
\hline miR-1284 & 2.084 & 0.049 & Up \\
miR-542-3p & 3.159 & 0.027 & Up \\
miR-877-3p & 3.216 & 0.045 & Up \\
miR-643 & 6.209 & 0.006 & Up \\
miR-3943 & 2.100 & 0.023 & Up \\
miR-377-5p & 0.347 & 0.038 & Down \\
miR-548l & 0.340 & 0.038 & Down \\
miR-146b-5p & 0.224 & 0.006 & Down \\
miR-BART19-3p & 0.377 & 0.049 & Down \\
miR-26a-2-3p & 0.430 & 0.031 & Down \\
miR-455-3p & 0.487 & 0.045 & Down \\
miR-34a-5p & 0.360 & 0.008 & Down \\
miR-146a-5p & 0.104 & 0.003 & Down \\
\hline
\end{tabular}




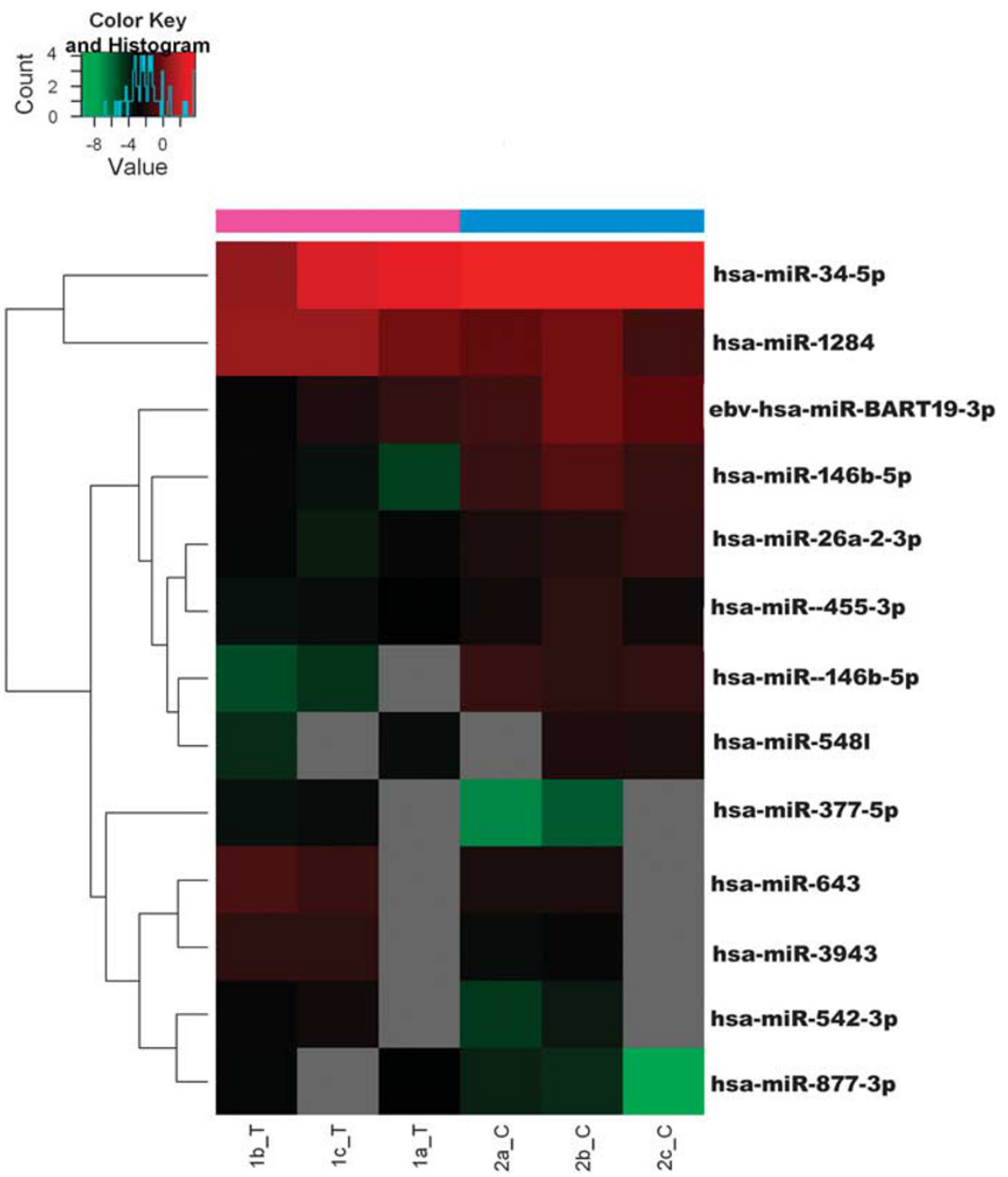

Figure 1 MiRNAs clustering analysis. MiRNA microarray analysis was used to examine the miRNA levels in MHCC97-H and MHCC97-L cell lines. Team T represented MHCC97-H cells and team C represented MHCC97-L cell line. Upregulated and downregulated miRNAs are shown in red and green colors, respectively. Thirteen miRNAs were identified to be significantly expressed, including five upregulated miRNAs (miR-1284, miR-542-3p, miR-877-3p, miR-643, miR-3943) and eight downregulated miRNAs (miR-377-5p, miR-548l, miR-146b-5p, ebv-miR-BARTA19-3p, miR-26a-2-3p, miR-455-3p, miR-34a-5p, miR-146a-5p), in MHCC97-H cells.

(3.96-fold), ebv-miR-BART19-3p (2.93-fold), miR-26a-2-3p (2.71-fold), miR-455-3p (1.82-fold), miR-34a-5p (3.14-fold), miR-146a-5p (7.87-fold) expression were lower in MHCC97$\mathrm{H}$ cell lines (Figure 2a). Evaluation of miRNA expression was also performed in HCC tissues and normal tumor-adjacent tissues, the result showed that miR-542-3p (2.80-fold), miR-877-3p (2.41-fold) and miR-643 (1.92-fold) were higher in HCC tissues, and miR-377-5p (1.72-fold), miR-26a-2-3p (2.05-fold), miR-455-3p (2.05-fold), miR-5481 (2.78-fold), miR-146b-5p (2.71-fold), miR-34a-5p (3.56-fold) and miR-146a-5p (2.05-fold) were higher in normal tumor-adjacent tissues. No significant change in miR-1284, miR-3943 and ebv-miR-BART19-3p was seen between HCC tissues and normal tumor-adjacent tissues (Figure 2b).

\section{Prediction and Validation of Target mRNA}

To further explore the relationship between miRNA and HCC metastasis, we utilized the online target gene prediction software programs miRanda, microCosma and targetscan to predict the target mRNA. Each miRNA has many target mRNAs; a single mRNA could also be regulated by more than one miRNA. 3'-UTR of ST3GAL5 contains putative recognition sites that could bind to the sequence of miR-26a, miR-548l and miR-34a, indicating the possible role of these three miRNAs in modulating ST3GAL5. The results of qRTPCR in 27 corresponding pairs of HCC tissues and normal tumor-adjacent tissues revealed that there were negative correlations between ST3GAL5 and miR-26a $(r=-0.3005$, $P=0.00273), \quad$ miR-5481 $\quad(r=-0.2956, \quad P=0.0300) \quad$ and 
a

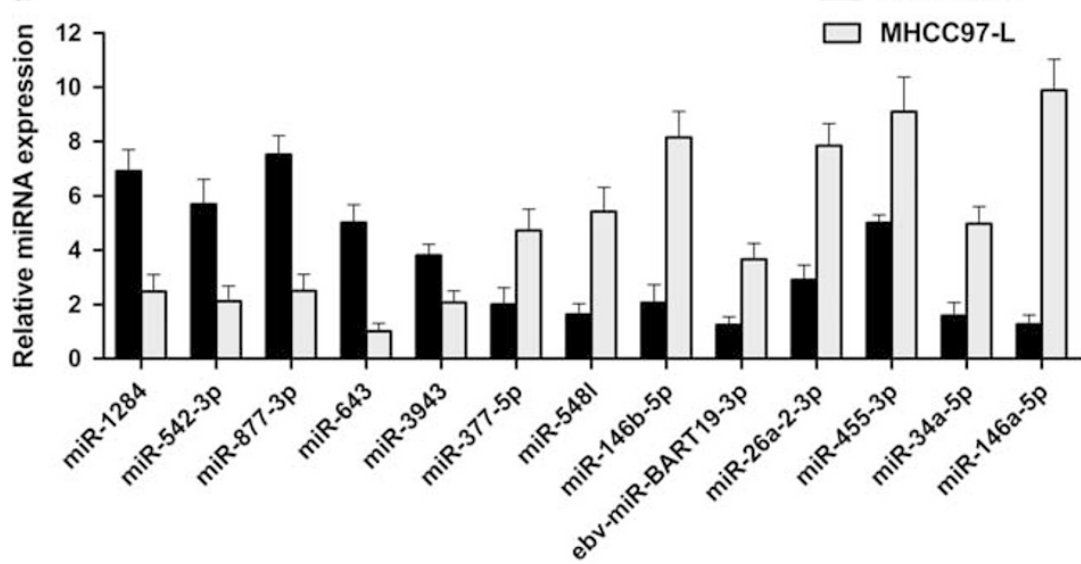

b

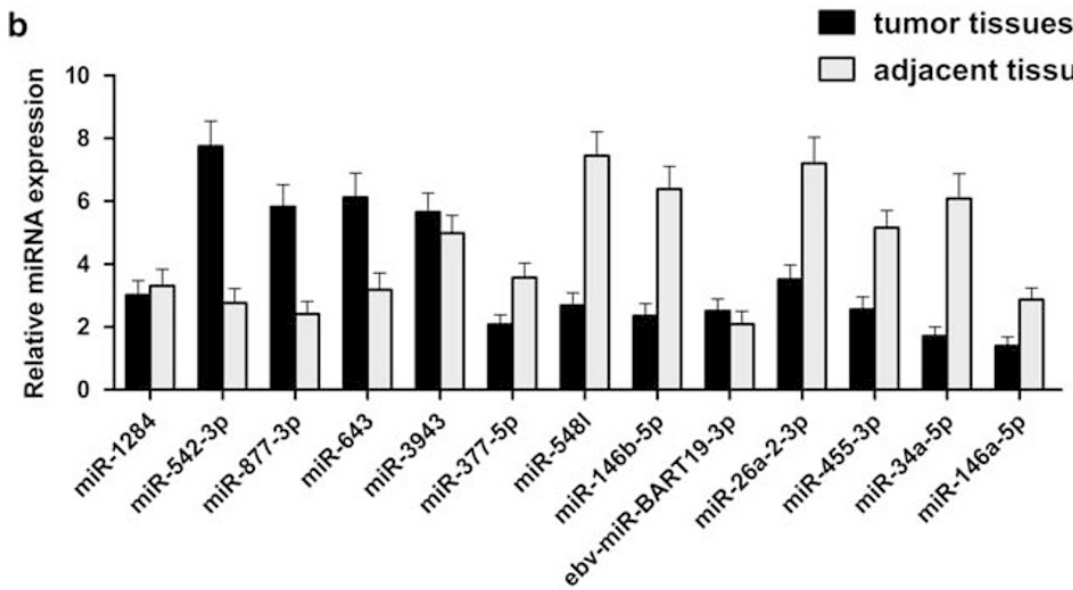

Figure 2 Thirteen miRNAs are differentially expressed in HCC cell lines and HCC tissues. (a) The miRNA levels were analyzed by qRT-PCR in two HCC cells. (b) Real-time PCR revealed that 13 miRNAs were also differentially expressed in HCC tissues compared with adjacent tissues. Data are the means \pm s.d. of triplicate determinants.

miR-34a $(r=0.5425, P<0.0001)$ (Figure 3a), and ST3GAL5 mRNA were highly expressed in HCC tissues in which the expression of miR-26a, miR-5481 and miR-34a were lower (Figure 3b).

To verify whether ST3GAL5 is the target mRNA of miR-26a, miR-5481 and miR-34a, we constructed $3^{\prime}$-UTR luciferase reporters with wild-type ST3GAL5 containing the potential three miRNA recognition elements, respectively, and corresponding mutated 3'-UTR reporters of ST3GAL5. A construct with renilla luciferase only acted as control. The results showed that the co-transfection of miR-26a, miR-5481 and miR-34a with ST3GAL5 wild-type reporter in HEK 293T cells displayed decreased luciferase activities compared with the NC groups, whereas luciferase activity of the mutant ST3GAL5 reporter changed little (Figures 3c-e). Furthermore, the results of qRT-PCR and western blotting showed that miR-26a, miR-548l and miR-34a mimics suppressed the expression of ST3GAL5, downregulated the expression of miR-26a, miR-548l and miR-34a and significantly improved the expression of ST3GAL5 at both mRNA and protein levels
(Figures $3 \mathrm{f}$ and $\mathrm{g}$ ). These results indicated that miR-26a, miR-5481 and miR-34a can directly regulate ST3GAL5.

\section{miR-26a, miR-548I and miR-34a Regulate HCC Progression In Vitro}

In order to explore whether and how miR-26a, miR-548l and miR-34a modulate HCC progression, we first overexpressed these three miRNA expression in MHCC97-H cells, and simultaneously downregulated the miRNA expression in MHCC97-L cells. Then CCK-8 assays, colony-forming assays and transwell assays were carried out to observe the effects of miR-26a, miR-5481 and miR-34a on HCC progression. Figure 4a shows that the expression of miR-26a, miR-5481 and miR-34a were strongly increased by their mimics. The results of the CCK- 8 assays and colony-forming assay reveal that overexpression of miR-26a, miR-5481, miR-34a and a mixture of these three miRNAs repressed cell proliferation (Figures $4 \mathrm{~b}$ and $\mathrm{c}$ ). The number of colony formed in miR-26a, miR-548l, miR-34a and miR mixture overexpressed groups were, respectively, $175 \pm 18,98 \pm 12,140 \pm 25$ and 
a

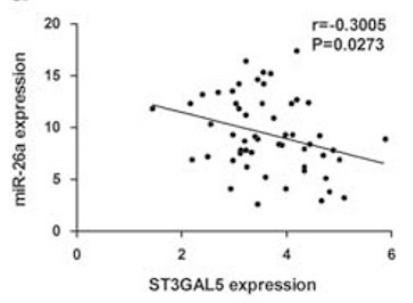

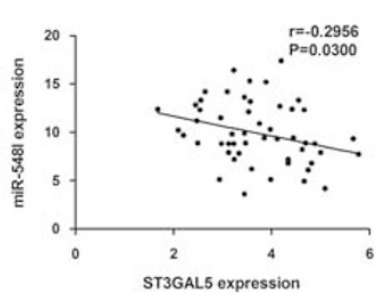

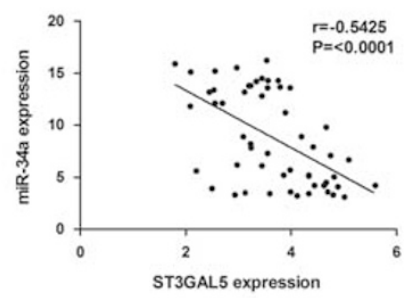

b

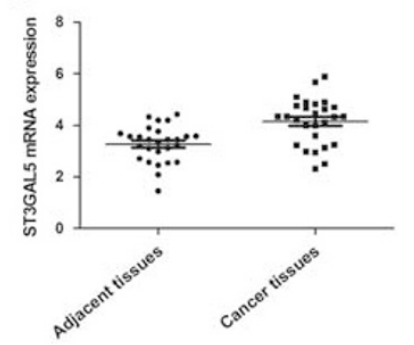

c

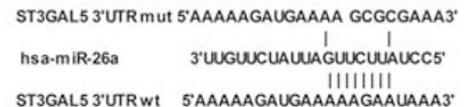

ST3GAL5 3'UTR wt 5'AAAAAGAUGAAAAIIIII

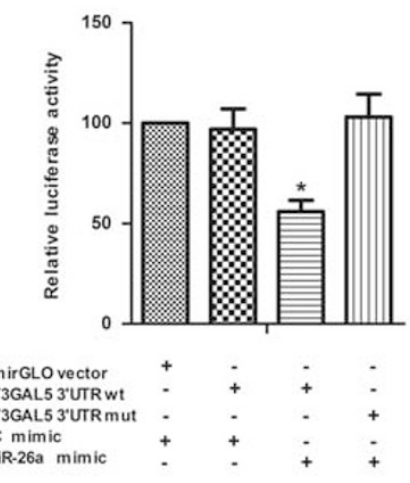

d

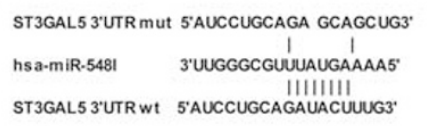

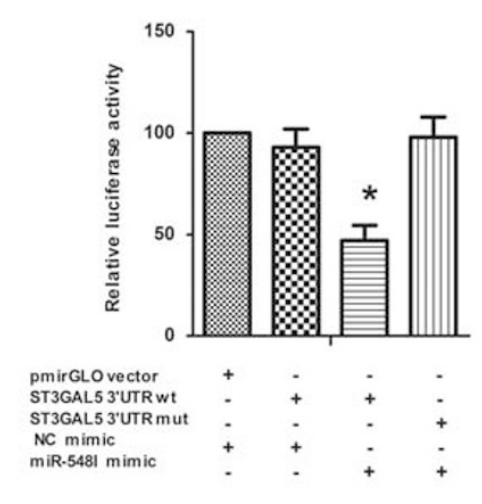

e

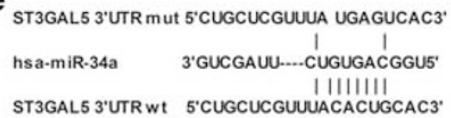
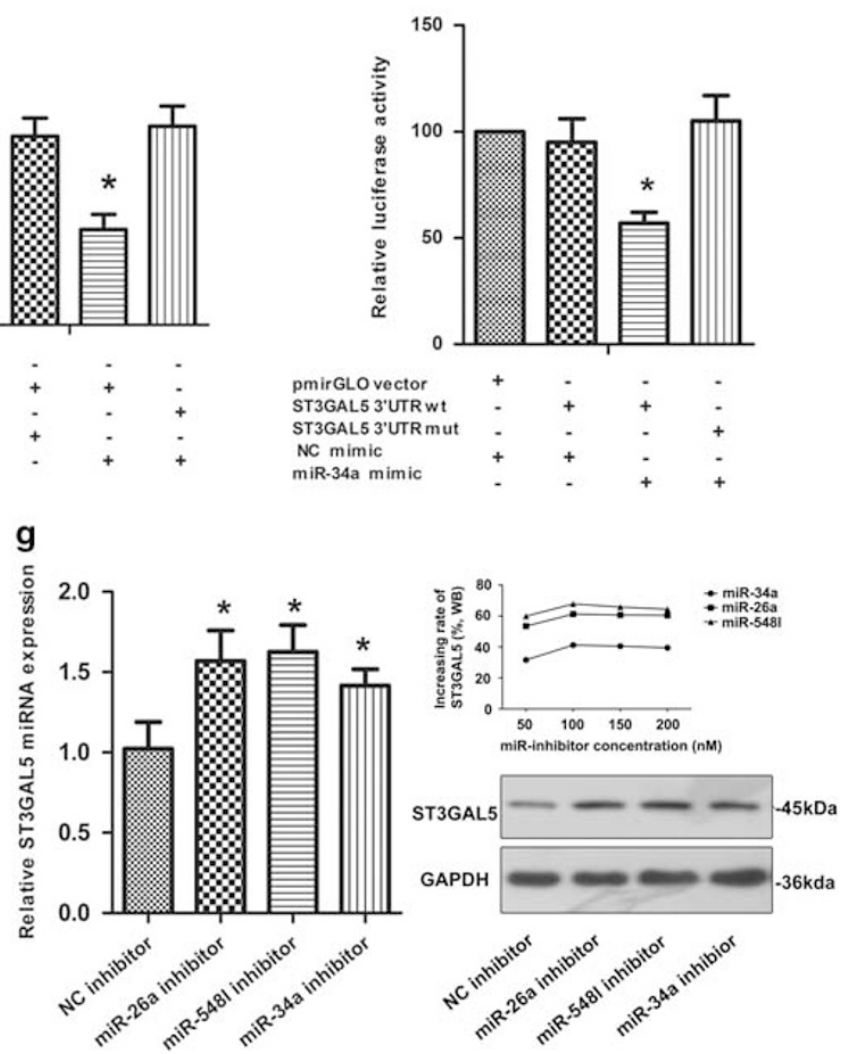

Figure 3 MiR-26a-2-3p, miR-548I and miR-34a-5p directly regulate ST3GAL5. (a) The correlation analyses between MiR-26a-2-3p/miR-548I/miR-34a-5p and ST3GAL5 expression using SPSS 13.0 statistical packages. (b) ST3GAL5 expression was significantly increased in 27 HCC tissues compared with the corresponding adjacent tissues using qRT-PCR analysis. (c-e) The luciferase assays confirmed that miR-26a-2-3p, miR-548I and miR-34a-5p bind to the wild-type 3'-UTR sequences of ST3GAL5. Mut: contained five-base mutation at miR-26a-2-3p, miR-548I and miR-34a-5p target region. The wide-type and mutant miRNA target sequences of ST3GAL5 were fused with luciferase reporter and transfected into $293 \mathrm{~T}$ cells, along with miRNA mimic or NC mimic. The mean of the results from the cells transfected with the NC mimic was set at 100. Each bar represents the relative luciferase activity. Co-transfection of miR-26a-2-3p, miR-548l or miR-34a-5p mimics with wide-type ST3GAL5 significantly reduced the luciferase levels (*P<0.05). (f) HCC cells were incubated with various concentrations of mimic (10,50, 100 and $200 \mathrm{nM})$ to find out the maximum effective concentration, and $100 \mathrm{nM}$ was determined to be the best concentration by western blotting analysis. qRT-PCR and western blotting revealed that ST3GAL5 expression in MHCC97-H cells were significantly decreased with treatment of $100 \mathrm{nM}$ miR-26a-2-3p, miR-548I and miR-34a-5p mimics $\left({ }^{*} P<0.05\right.$ vs group without an asterisk). (g) HCC cells were incubated with various concentrations of inhibitor (10,50, 100 and $200 \mathrm{nM})$ to find out the maximum effective concentration, and $100 \mathrm{nM}$ was determined to be the best concentration by western blotting analysis. The mRNA and protein levels of ST3GAL5 in MHCC97-L cells were dramatically increased with section of miR-26a-2-3p, miR-548I and miR-34a-5p inhibitors ( ${ }^{*} P<0.05$ vs group without an asterisk). Data are the means \pm s.d. of triplicate determinants. 
a
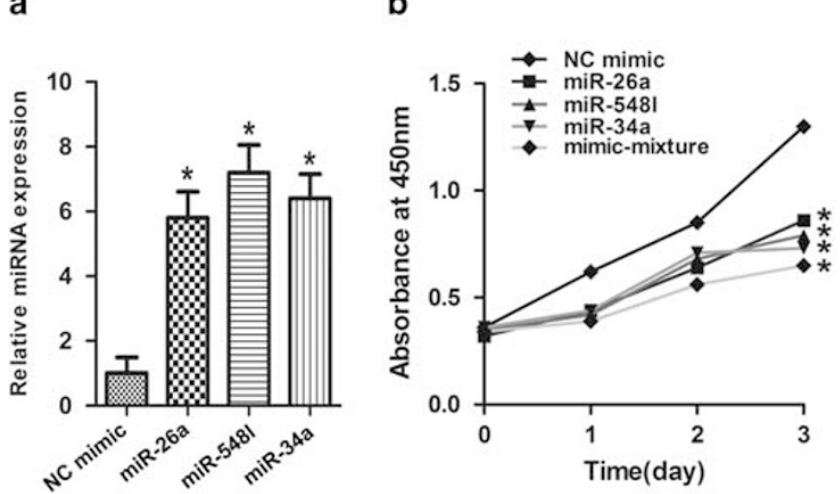

C
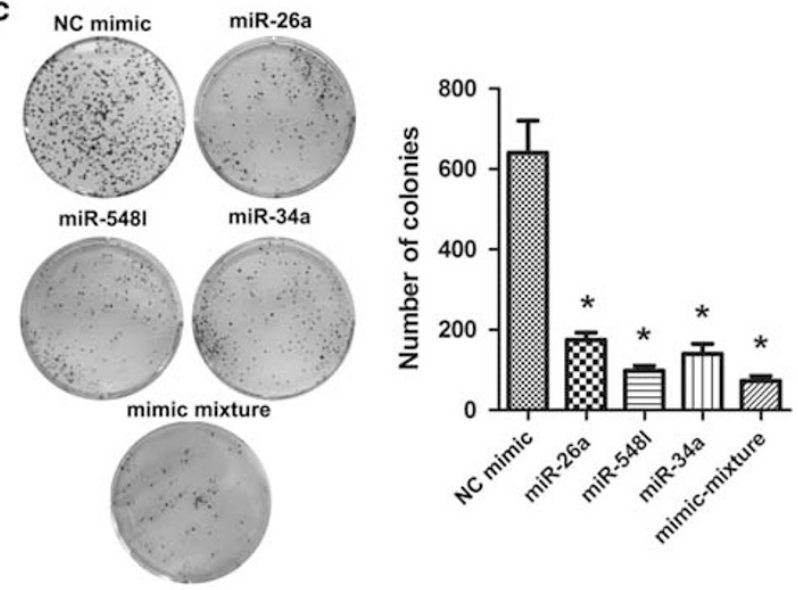

d
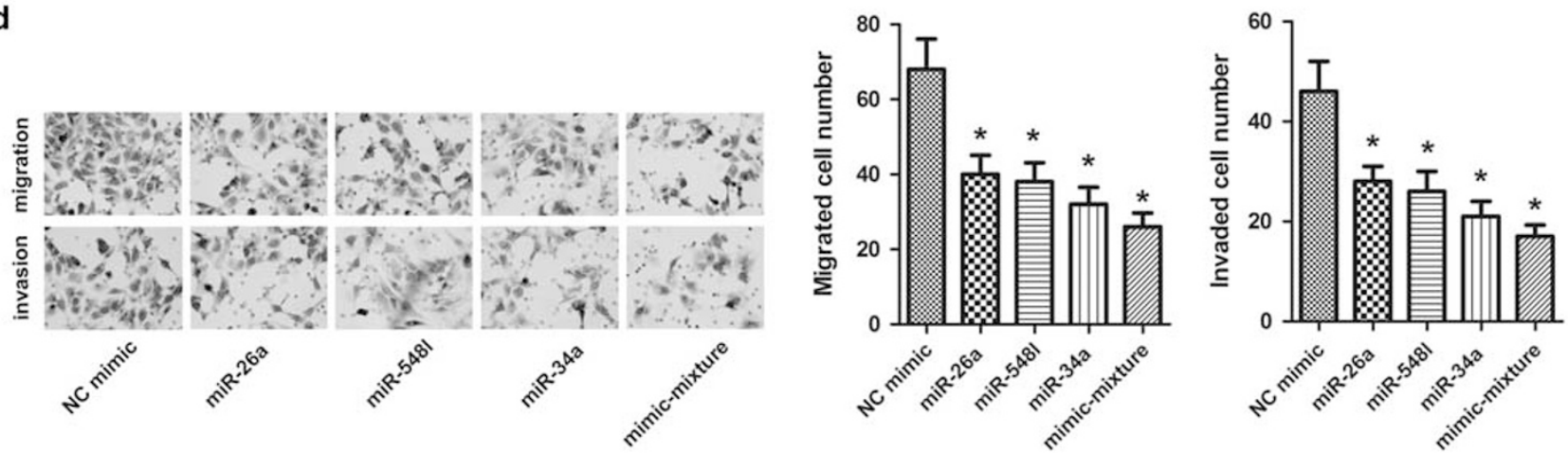

Figure 4 miR-26a-2-3p, miR-548I and miR-34a-5p mimics suppress MHCC97-H cells progression. (a) The expression of miR-26a-2-3p, miR-548I and miR-34a-5p in MHCC97-H cells were significantly increased with transfection of miRNA mimics ( ${ }^{*} P<0.05$ vs group without an asterisk). (b) CCK-8 assay revealed that treatment of miR-26a-2-3p, miR-548I, miR-34a-5p and miR-mixture mimics in MHCC97-H cells blocked cellular viability (* $P<0.05)$.

(c) Overexpression of miR-26a-2-3p, miR-548I or miR-34a-5p in MHCC97-H cells inhibited cells' growth as shown by colony-forming assay. (d) Transwell assays were performed to compare cell migration and invasion capacity. Ability of migration and invasion of cells transfected with miRNA mimics was significantly suppressed. The data were mean \pm s.d. of three separate transfections ( ${ }^{*} P<0.05$ vs group without an asterisk).

$75 \pm 8$, which are significantly less than the control group, $640 \pm 80(P<0.05)$. Additionally, the migration and invasion abilities of MHCC97-H cells were also inhibited by overexpression of miR-26a, miR-548l, miR-34a and miR mixture (Figure 4d). Co-transfection of three miRNAs showed a stronger effect than any of them alone.

Conversely, knockdown of miR-26a, miR-5481 and miR-34a promoted cell proliferation, migration and invasion. As shown in Figure 5a, significant inhibition was seen in MHCC97-L cells with anti-miR-26a, miR-5481 and miR-34a transfection. CCK-8 assay and colony-forming assay revealed that the short- and long-term proliferative ability in cells transfected with inhibitors of miR-26a, miR-548l, miR-34a and the mixture were higher than that of controls (Figures $5 \mathrm{~b}$ and $\mathrm{c}$ ). Transwell assays showed that downregulated expression of miR-26a, miR-5481 and miR-34a in MHCC97-L cells could enhance migration and invasion abilities significantly (Figure $5 \mathrm{~d}$ ). Thus a synergistic effect was seen in HCC cells with co-treatment of three miRNA inhibitors.

\section{Engineered Expression of miRNAs Modulate Tumor Growth In Vivo}

To further assess the relationship between miRNAs and HCC progression, tumor xenograft models were used. MHCC97-H cells that had been transfected with miR-26a, miR-5481, miR-34a, miR mixture mimics and NC mimics or miR-26a, miR-548l, miR-34a and miR mixture inhibitors were injected subcutaneously into the right flank of each nude mouse, respectively. Tumor size was observed and measured weekly after tumor was formed. The data revealed that tumor size and weight in the miR-26a, miR-5481, miR-34a and miR mixture mimics groups were significantly smaller and less than that of the NC group (Figure 6a). ST3GAL5 protein level in tumor tissue was reduced with miR-26a, miR-5481, miR-34a and miR mixture treatment by IHC analysis (Figure 6b). Figure 6c shows that tumor size and weight in each miR inhibitor group were significantly increased compared with that of the NC group. Figure 6d illustrates the levels of upregulation of ST3GAL5 protein in tumor tissue with miR inhibitor transfection. 


\section{ST3GAL5 Regulates HCC Progression}

As ST3GAL5 is the target of miR-26a, miR-548l and miR-34a and miR-26a, miR-548l and miR-34a have been shown to correlate with HCC progression, we further investigated the underlying mechanisms and confirmed that miR-26a, miR-5481 and miR-34a regulated HCC progression by targeting ST3GAL5.

As Figure 7a shows, ST3GAL5 was highly expressed in MHCC97-H cells than that of MHCC97-L cells. Overexpression and knockdown of ST3GAL5 successfully altered the expression of ST3GAL5 in MHCC97-L and MHCC97-H cells (Figures $7 \mathrm{~b}$ and e). CCK-8 assays revealed that, with the transfection of ST3GAL5 in MHCC97-L cells, the absorbance was increased over that of the control groups, indicating that overexpression of ST3GAL5 improved the proliferative ability of HCC cells (Figure 7c). In contrast, knockdown of ST3GAL5 repressed the cells' proliferative rate (Figure 7f). Furthermore, the results of transwell assays show that upregulation of ST3GAL5 significantly promoted the migratory and invasive ability of MHCC9-L cells (Figure 7d), and

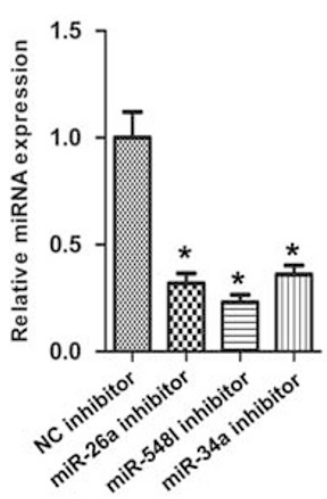

b

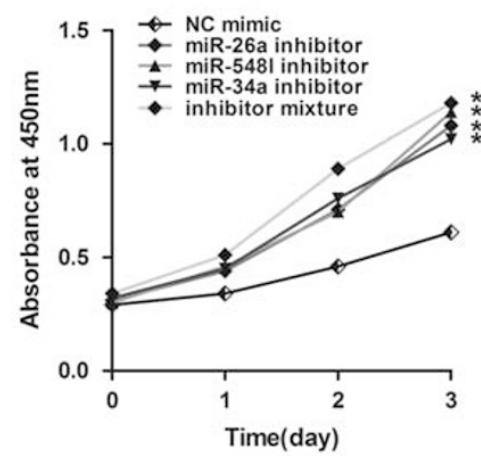

C
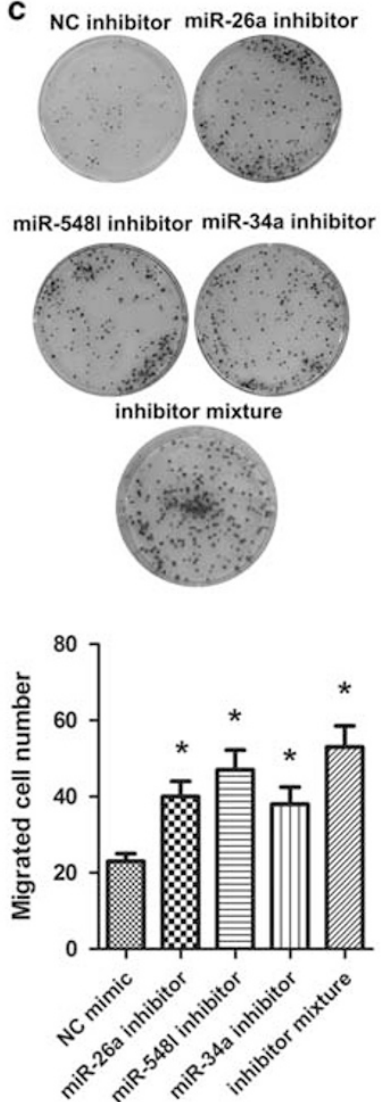
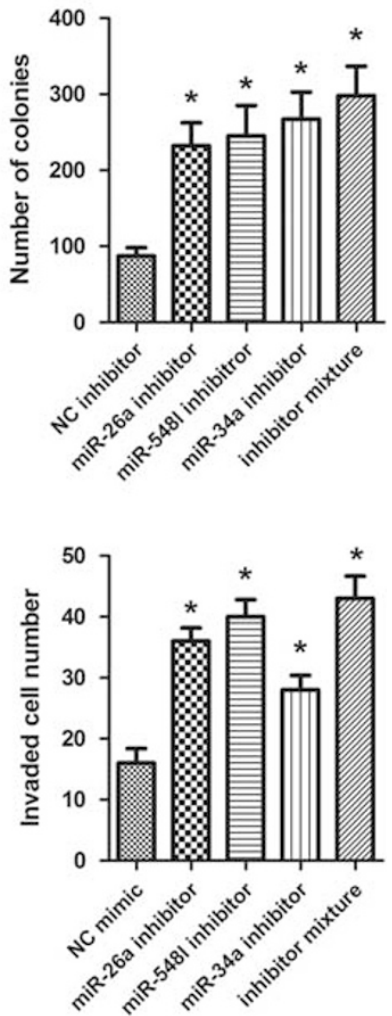

Figure 5 miR-26a-2-3p, miR-548I and miR-34a-5p inhibitors facilitate MHCC97-L cell progression. (a) The inhibition of miR-26a-2-3p, miR-548I and miR-34a-5p in MHCC97-L cells were confirmed by qRT-PCR ( ${ }^{*} P<0.05$ vs group without an asterisk). (b) CCK-8 assay showed that transfection of miR-26a2-3p, miR-548I, miR-34a-5p or miR-mixture inhibitor in MHCC97-L cells promoted cell viability $\left({ }^{*} P<0.05\right.$ vs group without an asterisk). (c) The colonies' number of MHCC97-L cells was increased with treatment of miRNA inhibitors as revealed by colony-forming assay. (d) Transwell assays were used to analyze cell migration and invasion capacity. miRNA inhibitors enhanced the ability of migration and invasion of MHCC97-L cells. The data were mean \pm s.d. of three separate transfections $\left({ }^{*} P<0.05\right.$ vs group without an asterisk).

Figure 6 MiR-26a-2-3p, miR-548I and miR-34a-5p regulates MHCC97-H cells tumorigenesis in vivo. (a) miRNA mimics or NC mimic were transfected in MHCC97-H cells, and then cells were inoculated subcutaneously into the right flank of each nude mouse, respectively $(n=6)$. Overexpression of miR-26a2-3p, miR-548I and miR-34a-5p inhibited the tumor growth of MHCC97-H cells in vivo. The tumor volumes and weight were reduced in the miRNA mimic groups as compared with the NC mimic group $(* P<0.05$ vs group without an asterisk). (b) The expression levels of ST3GAL5 were analyzed by IHC in tumor sections. (c) miRNA inhibitors or control inhibitor were transfected in MHCC97-L cells, aand then cells were inoculated subcutaneously into the right flank of each nude mouse, respectively $(n=6)$. miR inhibitors enhanced the tumor growth of MHCC97-L cells in vivo. The tumor volumes and weight were increased in the miRNA inhibitor groups as compared with the control group $\left({ }^{*} P<0.05\right.$ vs group without an asterisk). (d) IHC analysis revealed ST3GAL5 expression in tumor sections. 
blocking of ST3GAL5 in MHCC97-H resulted in reduction of the percentage of migratory and invasive cells (Figure $7 \mathrm{~g}$ ). Taken together, the above results demonstrate that miR-26a, miR-5481 and miR-34a most likely mediated HCC progression by targeting ST3GAL5.

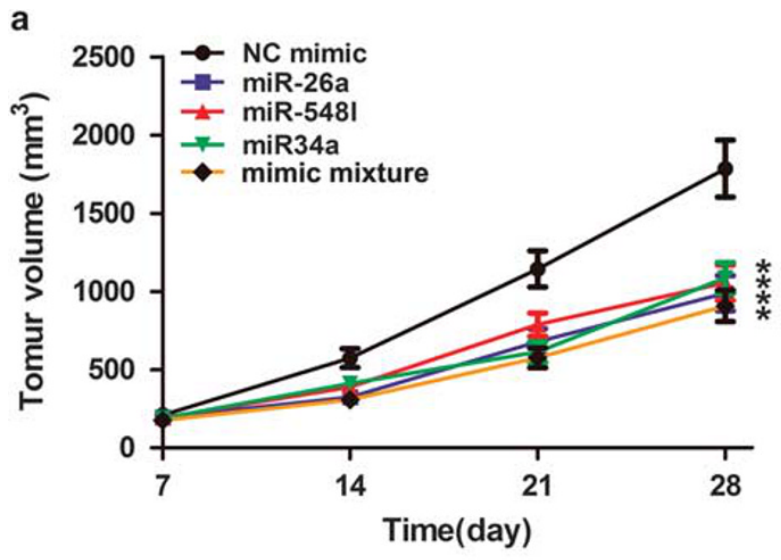

\section{DISCUSSION}

HCC is one of the most common cancers of the digestive tract. The annual incidence of HCC around the world is estimated to be $600000 .{ }^{17}$ Current therapeutic strategies for advanced HCC offer only limited efficacy. Early metastases

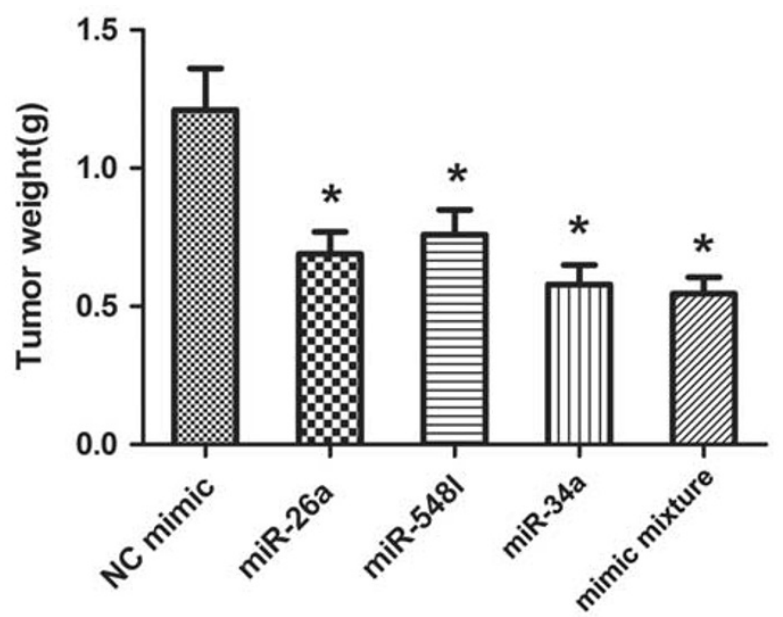

b

ST3GAL5

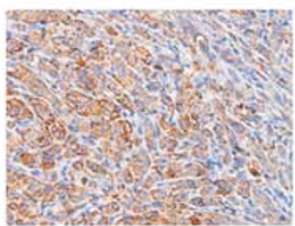

NC mimic

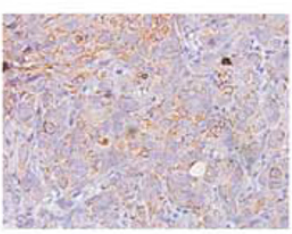

miR-26a

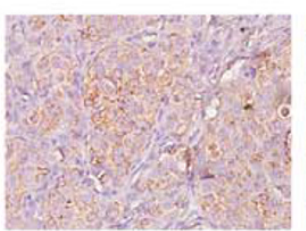

miR-548I

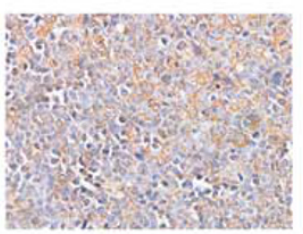

miR-34a

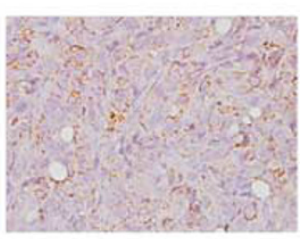

mimic mixture
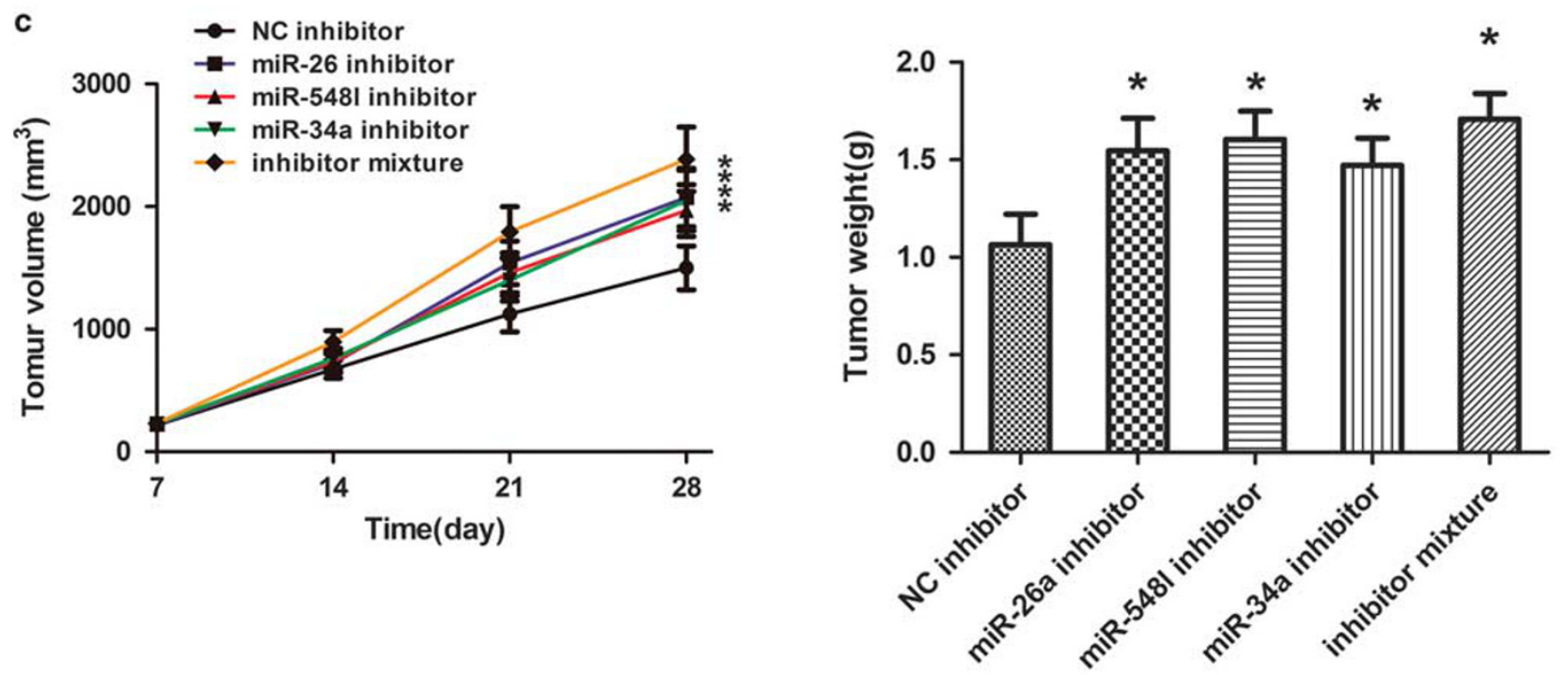

d

ST3GAL5

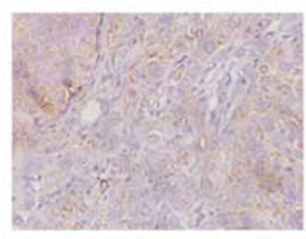

NC inhibitor

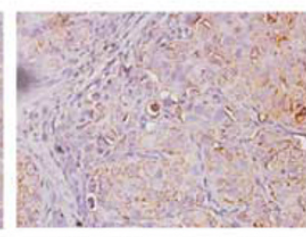

miR-26a inhibitor

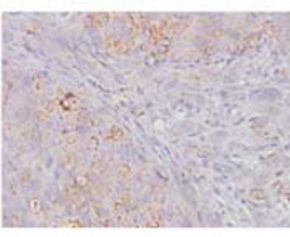

miR-548I inhibitor

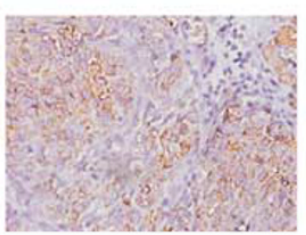

miR-34a inhibitor

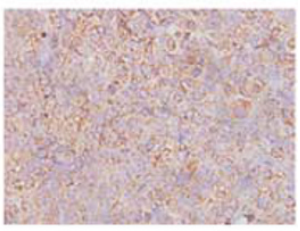

inhibitor mixture 

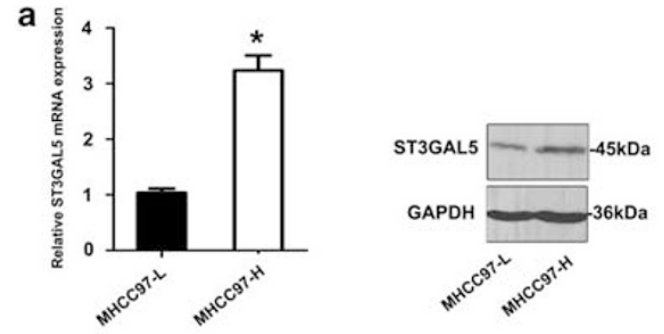

b
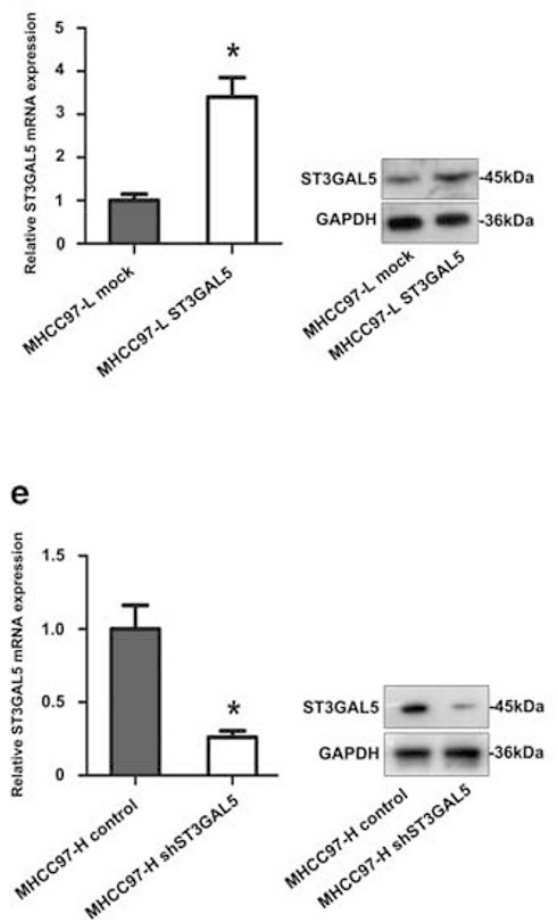

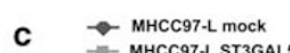

C MHCC97-LST3GAL5

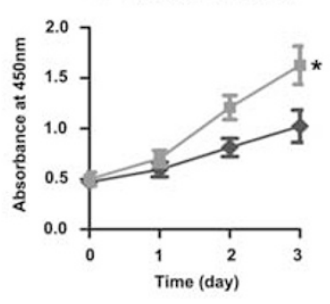

d

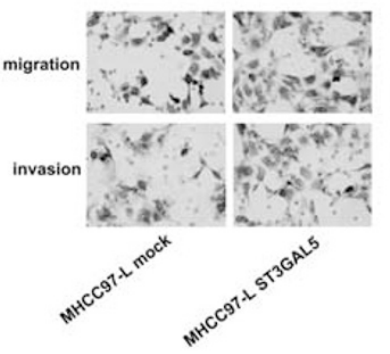

f $\rightarrow$ MHCC97-H control

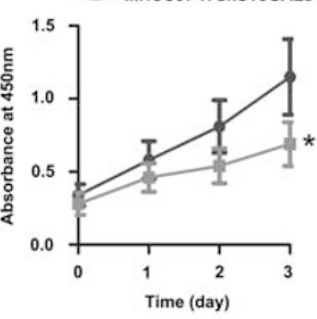

g

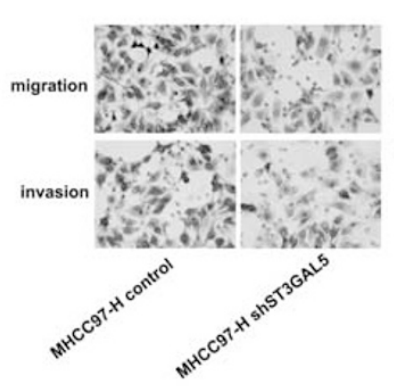

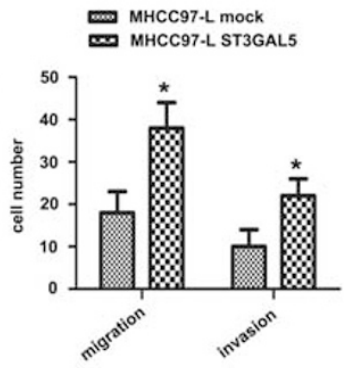

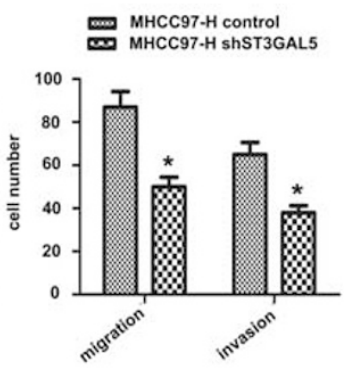

Figure 7 ST3GAL5 mediates HCC cells progression in vitro. (a) ST3GAL5 levels between high and low metastatic HCC cell lines (MHCC97-H and MHCC97-L) were determined by qRT-PCR and western blotting analysis. (b) The mRNA and protein levels of ST3GAL5 were increased notably with upregulation of ST3GAL5 in MHCC97-L cells as revealed by qRT-PCR and western blotting analysis. (c) CCK-8 assay showed growth curves of MHCC97L-ST3GAL5 cells and control cells. (d) Transwell analysis was carried out. MHCC97-L-ST3GAL5 cells were significantly more migratory and invasive than MHCC97-L mock cells. (e) ST3GAL5 mRNA and protein levels were decreased apparently in MHCC97-H cells with shRNA treatment as showed by qRT-PCR and western blotting analysis. (f) CCK-8 assay was performed to compare cellular viability between MHCC97-H-shST3GAL5 cells and control cells. (g) Transwell assays were conducted to compare cell migration and invasion between MHCC97-H-shST3GAL5 cells and the control group. *Indicates statistical difference compared with control $(P<0.05)$.

are the major cause of death of HCC patients. Therefore, intensive understanding of the molecular biomarkers and underlying mechanisms for HCC progression and metastasis is necessary.

Metastasis is a complex process regulated by multiple factors. As the relationship between cancer and microRNAs was first reported in $2002,{ }^{18}$ there is ample evidence of the involvement of miRNAs in promoting or inhibiting aggressive behavior of multiple types of cancer cells. In the present study, we used microarray technology to define differentially expressed miRNA profiles that are potent modulators of HCC invasiveness in two human HCC cell lines, MHCC97-H and MHCC97-L, which have high and low potentials of metastasis. A total of 13 genes that exhibited different levels were revealed. There were five upregulated miRNAs (miR-1284, miR-542, miR-877, miR-643, miR-3943) and eight downregulated miRNAs (miR-377, miR-548l, miR-146b, ebv-miR-BARTA19, miR-26a, miR-455, miR-34a, miR-146a) in MHCC97-H cells when compared with MHCC97-L cells. qRT-PCR also confirmed the microarray data in two HCC cell lines and 27 paired HCC and adjacent samples. Among these miRNAs, the involvement of miR-34a in hepatocarcinoma has been reported in the literature. MiR-34a was significantly downregulated in HCC tissues 
compared with adjacent non-tumor tissues. ${ }^{19,20}$ It has been determined that miR-26a is associated with an increased risk of hepatitis B virus-related $\mathrm{HCC}^{21}$ and downregulated in HCC tissues. ${ }^{22}$ MiR-146a was frequently downregulated in HCC and associated with tumor invasion and metastasis. ${ }^{23}$ MiR-146b was upregulated in five different drug-resistant HCC sublines. ${ }^{24}$ In addition, downregulation of miR-377 and miR-455 were observed in HCC tissues and cell lines, and miR-377 could dramatically inhibit cell growth and invasion in HCC cells. ${ }^{25,26}$ MiR-877 was also upregulated in paclitaxelinduced HCC cells, and overexpression of miR-877 in HCC cells partially restored paclitaxel sensitivity. ${ }^{27}$ On the other hand, the functions of miR-1284, miR-542, miR-643, miR-3943, miR5481 and ebv-miR-BART19 in HCC pathobiology is not well understood. This screen partially validated findings from previous researches, as miRNAs appear to have opposing effects on tumor growth in different types of cancer cells, serving as either a tumor suppressor or an oncogene. The miRNA expression profiles in MHCC97-H and MHCC97-L cell lines are important findings in the search for new candidate genes related to metastasis of HCC.

Identifying target genes may help us better understand the function of miRNAs. Bioinformatics methods revealed that miR-34a, which is well known for its role in cancer progression, is associated with HCC metastasis through direct regulation of the ST3GAL5 gene by binding to its $3^{\prime}$-UTR. Interestingly, miR-26a and miR-5481 also contains putative regions that could bind to the 3 '-UTR of ST3GAL5, indicating that miR-26a, miR-548l and miR-34a have the same target mRNA. We hypothesized that, if any regulatory relationship exists between miRNA expression and metastasis, it is quite possible that abnormally expressed miRNAs contribute to the process of HCC metastasis. This study reveals that the engineered expression of miR-26a, miR-548l and miR-34a affected the proliferation and invasion abilities of two HCC cells by both in vitro and in vivo analyses. Less is known about the involvement of miR-5481 in metastasis than of miR-34a and miR-26a, and here we have determined that changes in miR-5481 levels led to altered metastatic ability of HCC cells.

Various sialic acids commonly decorate cell surface glycolipids and glycoproteins. The linkage of sialic acid to $\mathrm{N}$-glycans occurs on the 3 or 6 position of galactose residues, termed $\alpha 2,3$ or $\alpha 2,6$ linkages, catalyzed by the ST3Gal and ST6Gal enzyme families, respectively. ${ }^{28,29}$ In some tumors, the level of sialylation on the cell surface may serve as a biomarker of the degree of malignancy. ${ }^{30}$ Lee et al ${ }^{31}$ reported that $\alpha 2,3$ sialic acid expression increased on the liver membrane of tumor-bearing mice, reflecting the upregulated levels of $\alpha 2,3 \mathrm{ST}$ and the lectin receptor. Our previous study found that the composition profiling of sialylated $\mathrm{N}$-glycans differed in two HCC cell lines with different metastatic potential by using mass spectrometric analysis, which implies that there is a role for sialylation in the regulation of invasion and metastasis of HCC. ${ }^{32}$ In this study, we observed that
ST3GAL5 overexpression significantly promoted survival and invasive ability of MHCC97-L cells; by contrast, ST3GAL5 knockdown inhibited proliferation and metastasis of MHCC97-H cells. These results indicate the substantial association of ST3GAL5 with HCC metastasis. Several members of ST3Gal family have been demonstrated to be tumor promoters, for example, ST3Gal I was shown to be involved in the invasiveness and metastatic nature of HCC cells and might be an independent predictor of prognosis and the appropriate pharmaceutical target in patients with HCC. ${ }^{33}$ Knockdown of ST3GAL6, another member of ST3Gal family, led to a significant reduction in the levels of $\alpha 2,3$-linked sialic acid on the surface of multiple myeloma cells, along with reduced transendothelial migration in vitro and decreased tumor size and prolonged survival in vivo. ${ }^{34}$ In the current study, we also found that HCC samples from patients had higher ST3GAL5 expression than that of adjacent tissues, and this could further confirm our findings in vitro. Abnormal expression of ST3Gal5 is found in several types of cancers, but little is known about the underlying mechanisms of its involvement in human HCC. On the basis of our results, we propose that ST3GAL5 may contribute to the initial metastatic step in HCC by regulating the levels of sialylated $\mathrm{N}$-glycans and further affect cell adhesion and migration, leading to a worse prognosis. ST3GAL5, together with its upstream miRNAs' regulator, may be the risk factor for the HCC-free survival. This might be suggestive that targeting ST gene could be a new strategy to deal with HCC in the future.

Taken together, our experiments show that the differentially expressed miRNAs in two HCC cells with high or low metastasis ability may be useful as biomarkers for HCC progression. We propose, therefore, that miR-26a, miR-5481 and miR-34a, along with their target gene ST3GAL5, may be suitable for designing molecular therapeutic approaches to HCC.

\section{ACKNOWLEDGMENTS}

This work was supported by grants from the National Natural Science Foundation of China (81271910 and 81472014) and the Natural Science Foundation of Liaoning Province, China (2014023043)

\section{DISCLOSURE/CONFLICT OF INTEREST}

The authors declare no conflict of interest.

1. Chen W, Zheng $R$, Zhang $S$, et al. Annual report on status of cancer in China, 2010. Chin J Cancer Res 2014;26:48-58.

2. Torre LA, Bray F, Siegel RL, et al. Global cancer statistics, 2012. CA Cancer J Clin 2015;65:87-108.

3. London WT, McGlynn KA. Liver cancer. In: Schottenfeld D, Fraumeni Jr J (eds). Cancer Epidemiology and Prevention 3rd (edn).Oxford University Press: New York, USA, 2006;763-786.

4. Pang RW, Joh JW, Johnson PJ, et al. Biology of hepatocellular carcinoma. Ann Surg Oncol 2008;15:962-971.

5. Zhou ZJ, Dai Z, Zhou SL, et al. HNRNPAB induces epithelialmesenchymal transition and promotes metastasis of hepatocellular carcinoma by transcriptionally activating SNAIL. Cancer Res 2014;74: 2750-2762.

6. Baek D, Villén J, Shin $C$, et al. The impact of microRNAs on protein output. Nature 2008;455:64-71. 
7. Di Leva G, Garofalo M, Croce CM. MicroRNAs in cancer. Annu Rev Pathol 2014;9:287-314.

8. Shao Y, Li P, Zhu ST, et al. MiR-26a and miR-144 inhibit proliferation and metastasis of esophageal squamous cell cancer by inhibiting cyclooxygenase-2. Oncotarget 2016;7:15173-15186.

9. Qiu X, Zhang J, Shi W, et al. Circulating microRNA-26a in plasma and its potential diagnostic value in gastric cancer. PLos ONE 2016;11: e0151345.

10. Liu C, Yang $\mathrm{H}, \mathrm{Xu} \mathrm{Z}$, et al. MicroRNA-548l is involved in the migration and invasion of non-small cell lung cancer by targeting the AKT1 signaling pathway. J Cancer Res Clin Oncol 2015;141:431-441.

11. Zhang $X$, Liu $S$, Hu T, et al. Up-regulated microRNA-143 transcribed by nuclear factor kappa B enhances hepatocarcinoma metastasis by repressing fibronectin expression. Hepatology 2009;50:490-499.

12. Budhu $A$, Jia $H L$, Forgues $M$, et al. Identification of metastasis related microRNAs in hepatocellular carcinoma. Hepatology 2008;47:897-907.

13. Reis $\mathrm{CA}$, Osorio $\mathrm{H}$, Silva $\mathrm{L}$, et al. Alterations in glycosylation as biomarkers for cancer detection. J Clin Pathol 2010;63:322-329.

14. Lepers AH, Ruiz VV, Recchi MAK, et al. The human sialyltransferases family. Biochimie 2001;83:727-737.

15. Mondal A, Chandra S, Mandal C. Elevated mRNA level of hST6Gal I and hST3Gal V positively correlates with the high risk of pediatric acute leukemia. Leuk Res 2010;34:463-470.

16. Pérez-Garay $M$, Arteta $B$, Pagès $L$, et al. alpha2,3-sialyltransferase ST3Gal III modulates pancreatic cancer cell motility and adhesion in vitro and enhances its metastatic potential in vivo. PLOS ONE 2010;5: e12524.

17. Cabibbo G, Enea $M$, Attanasio $M$, et al. A meta-analysis of survival rates of untreated patients in randomized clinical trials of hepatocellular carcinoma. Hepatology 2010;51:1274-1283.

18. Calin GA, Dumitru CD, Shimizu M, et al. Frequent deletions and downregulation of micro- RNA genes miR15 and miR16 at 13q14 in chronic lymphocytic leukemia. Proc Natl Acad Sci USA 2002;99:15524-15529.

19. Chamani F, Sadeghizadeh M, Masoumi M, et al. Evaluation of miR-34 family and DNA methyltransferases 1, 3A, 3B gene expression levels in hepatocellular carcinoma following treatment with dendrosomal nanocurcumin. Asian Pac J Cancer Prev 2016;17:219-224.

20. Cui $X, W u Y$, Wang $Z$, et al. MicroRNA-34a expression is predictive of recurrence after radiofrequency ablation in early hepatocellular carcinoma. Tumour Biol 2015;36:3887-3893.

21. Wang C, Hann HW, Ye Z, et al. Prospective evidence of a circulating microRNA signature as a non-invasive marker of hepatocellular carcinoma in HBV patients. Oncotarget 2016 (doi:10.18632/oncotarget.9429).

22. Thurnherr T, Mah WC, Lei $Z$, et al. Differentially expressed miRNAs in hepatocellular carcinoma target genes in the genetic information processing and metabolism pathways. Sci Rep 2016;6:20065.

23. Zhang $Z$, Zhang $Y$, Sun $X X$, et al. microRNA-146a inhibits cancer metastasis by downregulating VEGF through dual pathways in hepatocellular carcinoma. Mol Cancer 2015;14:5.

24. Zhuo L, Liu J, Wang B, et al. Differential miRNA expression profiles in hepatocellular carcinoma cells and drug-resistant sublines. Oncol Rep 2013;29:555-562.

25. Chen G, Lu L, Liu C, et al. MicroRNA-377 suppresses cell proliferation and invasion by inhibiting TIAM1 expression in hepatocellular carcinoma. PLos ONE 2015;10:e0117714.

26. Chiu LY, Kishnani PS, Chuang TP, et al. Identification of differentially expressed microRNAs in human hepatocellular adenoma associated with type I glycogen storage disease: a potential utility as biomarkers. J Gastroenterol 2014;49:1274-1284.

27. Huang X, Qin J, Lu S. Up-regulation of miR-877 induced by paclitaxel inhibits hepatocellular carcinoma cell proliferation though targeting FOXM1. Int J Clin Exp Pathol 2015;8:1515-1524.

28. Okajima T, Fukumoto $\mathrm{S}$, Miyazaki $\mathrm{H}$, et al. Molecular cloning of a novel alpha2,3-sialyltransferase (ST3Gal VI) that 617 sialylates type II lactosamine structures on glycoproteins and glycolipids. J Biol Chem 1999;274:11479-11486.

29. Schauer R. Achievements and challenges of sialic acid research. Glycoconj J 2000;17:485-499.

30. Ellies LG, Sperandio M, Underhill GH, et al. Sialyltransferase specificity in selectin ligand formation. Blood 2002;100:3618-3620.

31. Lee A, Kolarich D, Haynes PA, et al. Rat liver membrane glycoproteome: enrichment by phase partitioning and glycoprotein capture. J Proteome Res 2009;8:770-781.

32. Zhao $\mathrm{Y}, \mathrm{Li} \mathrm{Y}, \mathrm{Ma} \mathrm{H}$, et al. Modification of sialylation mediates the invasive properties and chemosensitivity of human hepatocellular carcinoma. Mol Cell Proteomics 2014;13:520-536.

33. Wu H, Shi XL, Zhang HJ, et al. Overexpression of ST3Gal-I promotes migration and invasion of HCCLM3 in vitro and poor prognosis in humanhepatocellular carcinoma. Onco Targets Ther 2016;9: 2227-2236.

34. Glavey SV, Manier S, Natoni A, et al. The sialyltransferase ST3GAL6 influences homing and survival in multiple myeloma. Blood 2014;124: $1765-1776$. 